\title{
AN ALGEBRAIC DUALIZATION OF FUNDAMENTAL GROUPS
}

\author{
BY KUO-TSAI CHEN 1
}

Communicated by Saunders MacLane, March 17, 1969

This note presents a construction of a Hopf algebra $\pi^{1}(A)$ for a given augmented commutative algebra $A$ equipped with a derivation. Such a Hopf algebra may be taken as a dualized algebraic analogy of a fundamental group.

1. The construction of $\pi^{1}(A)$ is motivated by dualizing the fundamental group $\pi_{1}(X)$ of a differentiable manifold $X$ with a base point $x_{0}$. Let $A$ be the $R$-algebra of $C^{\infty}$ functions on $X$ equipped with the derivation $d$, which is the usual differentiation from $A$ into the $A$ module $M=\Omega A$ of $C^{\infty} 1$-forms on $X$. Recall that the shuffle algebra $\operatorname{Sh}(M)$ consists of the $R$-module of the tensor algebra $T_{R}(M)$ and the shuffle multiplication $\circ$. We make $\operatorname{Sh}(M)$ a Hopf $R$-algebra with the comultiplication $\zeta: \operatorname{Sh}(M) \rightarrow \operatorname{Sh}(M) \otimes \operatorname{Sh}(M)$ given by

$$
w_{1} \otimes \cdots \otimes w_{r} \mapsto \sum_{0 \leqq i \leqq r}\left(w_{1} \otimes \cdots \otimes w_{i}\right) \otimes\left(w_{i+1} \otimes \cdots \otimes w_{r}\right)
$$

$\forall w_{1}, \cdots, w_{r} \in M$. Moreover the Hopf algebra $\operatorname{Sh}(M)$ possesses an antipode (or conjugation) $j$.

Denote by $G$ the monoid of piecewise smooth loops of $X$ with the base point $x_{0}$ under the equivalence relation of reparametrization. The monoid algebra $R G$ is a Hopf algebra whose comultiplication $\Delta$ is given by $\Delta \alpha=\alpha \otimes \alpha, \forall \alpha \in G$.

Given a loop $\alpha:[0,1] \rightarrow X$, let $\int_{\alpha} w_{1}$ be the usual integral, and define, for $r>1$, iterated path integrals

$$
\int_{\alpha} w_{1} \cdots w_{r}=\int_{0}^{1}\left(\int_{\alpha \mid[0, t]} w_{1} \cdots w_{r-1}\right) w_{r}(\alpha(t), \dot{\alpha}(t)) d t .
$$

Then there is a pairing $\operatorname{Sh}(M) \times R G \rightarrow R$ such that

$$
\left\langle w_{1} \otimes \cdots \otimes w_{r}, \alpha\right\rangle=\int_{\alpha} w_{1} \cdots w_{r}
$$

1 Work supported in part by the National Science Foundation under Grant NSFGP-8500. 
This is actually a pairing of Hopf algebras so that, $\forall u, v \in \operatorname{Sh}(M)$, $\forall \alpha, \beta \in G$,

$$
\begin{aligned}
\langle u \circ v, \alpha\rangle & =\langle u, \alpha\rangle\langle v, \alpha\rangle=\langle u \otimes v, \Delta \alpha\rangle, \\
\langle u, \alpha \beta\rangle & =\langle\zeta u, \alpha \otimes \beta\rangle .
\end{aligned}
$$

Moreover

$$
\left\langle u, \alpha^{-1}\right\rangle=\langle j u, \alpha\rangle .
$$

In the above pairing, $\operatorname{Sh}(M)$ is too large and has an ideal $I$ which is orthogonal to $R G$. The ideal $I$ is spanned by elements of the type

$$
u \otimes f w \otimes v-(u \circ d f) \otimes w \otimes v-f\left(x_{0}\right) u \otimes w \otimes v,
$$

$\forall u, v \in \operatorname{Sh}(M), f \in A, w \in M$. (See [1].) Let $P(A)$ denote the augmented quotient $R$-algebra $\operatorname{Sh}(M) / I$. Set $\bar{u}=u+I$.

Observe that the 1 -form $d f \in M=T^{1}(M) \subset T(M)$ can be taken as an element of $\operatorname{Sh}(M)$ so that $\langle d f, \alpha\rangle=\int_{\alpha} d f=0$. Let $Q(A)$ denote the augmented quotient $R$-algebra of $P(A)$ over the ideal generated by all $\overline{d f}, f \in A$. It turns out that $Q(A)$ is a quotient Hopf $R$-algebra of $\operatorname{Sh}(M)$. The induced pairing $Q(A) \times R G \rightarrow R$ is again that of Hopf algebras.

Let $\alpha_{\varepsilon}, 0 \leqq s \leqq 1$, represent a piecewise smooth homotopy of loops from the loop $\alpha_{0}$ to the loop $\alpha_{1}$ at the base point $x_{0}$. Our interest is in those elements $u$ of $Q(A)$ such that $\left\langle u, \alpha_{s}\right\rangle$ is independent of $s$. For this purpose, we devise algebraically a derivation $\delta$ of the $R$ algebra $Q(A)$ such that, if $\delta u=0$, then

$$
(d / d s)\left\langle u, \alpha_{s}\right\rangle=(d / d s) \int_{\alpha_{s}} u=0 .
$$

Denote by $\pi^{1}(A)$ the kernel of the derivation $\delta$, which happens to be a Hopf $R$-algebra with an antipode. Obviously there is an induced pairing of Hopf algebras

$$
\pi^{1}(A) \times R \pi_{1}(X) \rightarrow R .
$$

In our construction described below, the algebra $P(A)$ will be, in addition equipped with a derivation.

2. All rings and algebras will be commutative and possessing one. Let $K$ be the ground ring. We begin with the category $C$ of $K$-modules and shall only make use of the fact that $C$ is a cocomplete tensored category, i.e. a tensored category (see [4]) which has tensor-productpreserving direct limits. 
Denote by $C^{\prime}$ the morphism category of $C$. An object $M$ of $C^{\prime}$ is a morphism of $K$-modules $d_{M}:|M| \rightarrow \Omega M$; and a morphism of $C^{\prime}, \phi$ : $M \rightarrow M^{\prime}$, consists of two morphisms of $K$-modules $|\phi|:|M| \rightarrow\left|M^{\prime}\right|$ and $\Omega \phi: \Omega M \rightarrow \Omega M^{\prime}$ with $d_{M^{\prime}}|\phi|=\Omega \phi d_{M}$. The tensor product $M \otimes_{c^{\prime}} M^{\prime}$ is defined to be

$$
\left(d_{M} \otimes 1,1 \otimes d_{M^{\prime}}\right):|M| \otimes\left|M^{\prime}\right| \rightarrow \Omega M \otimes\left|M^{\prime}\right| \oplus|M| \otimes \Omega M^{\prime} .
$$

Let $K^{\langle 1\rangle}$ denote the object $K \rightarrow 0$ in $C^{\prime}$. With the ground object $K^{\langle 1\rangle}$ and the tensor product $\otimes_{c^{\prime}}$, we have a cocomplete tensored category $C^{\prime}$. A $C^{\prime}$-algebra $A$ can be identified with a $K$-algebra $|A|$ equipped with a derivation $d_{A}:|A| \rightarrow \Omega A$. If $A$ is augmented, the augmentation of $A$ will be denoted by $\epsilon_{A}$.

3. A $C^{\prime}$-algebra $B$ is said to be exact if the sequence of $K$-modules

$$
0 \rightarrow K \rightarrow|B| \stackrel{d_{B}}{\rightarrow} \Omega B \rightarrow 0
$$

is exact. As in [1], we construct, for a given augmented $C^{\prime}$-algebra $A$, an augmented exact $C^{\prime}$-algebra $P(A)$ and a morphism of augmented $C^{\prime}$-algebras $\chi_{A}: A \rightarrow P(A)$ which is universal with respect to morphisms from $A$ to augmented exact $C^{\prime}$-algebras. Moreover, there is a quotient augmented $K$-algebra $Q(A)$ of $|P(A)|$ such that the projection $\rho:|P(A)| \rightarrow Q(A)$ is universal with respect to the property that $\rho\left|\chi_{A}\right|$ is the composition

$$
|A| \stackrel{\left|\epsilon_{A}\right|}{\longrightarrow} K \rightarrow Q(A) .
$$

It happens that $Q(A)$ is a Hopf $K$-algebra with commutative multiplication. (In [1], $P(A)$ and $Q(A)$ are respectively denoted by $\operatorname{Sh}(d, p)$ and $\operatorname{Shc}(d, p)$, where $d=d_{A}$ and $\left.p=\left|\epsilon_{A}\right|.\right)$

4. A $C^{\prime}$-algebra $A$ is said to be full if the composition

$$
|A| \otimes \operatorname{Im} d_{A} \rightarrow|A| \otimes \Omega A \stackrel{m}{\rightarrow} \Omega A
$$

is epic, where $m$ denotes the scalar multiplication of the $|A|$-module $\Omega A$.

Construct the cocomplete tensored category $C^{\prime \prime}=\left(C^{\prime}\right)^{\prime}$. Each object $M$ in $C^{\prime \prime}$ is then a square diagram in $C$ which can be transposed. The transposed object will be denoted by $M^{t}$.

Given an augmented full $C^{\prime}$-algebra $A$, there is an augmented $C^{\prime \prime}$-algebra $\{A\}$ which is universal with respect to the property that $|\{A\}|=|\{A\} t|=A$. 
Observe that the functors $P$ and $Q$ for the category $C^{\prime}$ can be similarly constructed for the category $C^{\prime \prime}$. Furthermore, $|Q(\{A\})|$ $=Q(A)$. Thus $Q(\{A\})$ represents a derivation $\delta$ of $Q(A)$.

We define $\pi^{1}(A)$ to be the kernel of the derivation $\delta$. If $\otimes$ is also left exact, then $\pi^{1}(A)$ is a Hopf subalgebra of $Q(A)$ and possesses an antipode.

ThEOREM. If $A$ is an augmented full $C^{\prime}$-algebra and if $d_{A}$ induces an exterior derivation $d_{1}: \Omega A \rightarrow \Omega A \Lambda_{|A|} \Omega A$, then the composite morphism of $K$-modules

$$
\Omega A \stackrel{\Omega \chi_{A}}{\longrightarrow}>\Omega P(A) \stackrel{i}{\rightarrow}|P(A)| \stackrel{\rho}{\rightarrow} Q(A)
$$

induces a morphism of $K$-modules

$$
\theta: H^{1}(A)=\operatorname{Ker} d_{1} / \operatorname{Im} d_{A} \rightarrow \pi^{1}(A),
$$

where $i$ is the morphism of $K$-modules which splits the exact sequence of the exact $C^{\prime}$-algebra $P(A)$ in the same way as the augmentation of $P(A)$ does.

REMark 1. If $A$ is the augmented $R$-algebra equipped with the derivation as described in $\$ 1$ and if the manifold $X$ is compact, then it follows from de Rham's theorem that $\theta$ is injective.

REMARK 2. If $X$ is a compact Riemannian surface, we let $K$ be the complex number field and denote by $A$ the augmented $K$-algebra of complex valued $C^{\infty}$ functions on $X$ equipped with the usual differentiation. Let $M^{\prime} \subset M=\Omega A$ be the $K$-module of abelian differentials of the first kind. It can be shown that the image of the composition

$$
\operatorname{Sh}\left(M^{\prime}\right) \rightarrow \operatorname{Sh}(M) \stackrel{\rho^{\prime}}{\rightarrow} Q(A)
$$

lies in $\pi^{1}(A)$, where $\rho^{\prime}$ denotes the projection. This can be roughly interpreted to mean that the Hopf algebra $\pi^{1}(A)$ contains a Hopf subalgebra consisting of iterated path integrals (from $x_{0}$ ) of abelian differentials of the first kind.

5. Two morphisms of augmented $C^{\prime}$-algebras $\phi_{i}: A \rightarrow A^{\prime}, i=0,1$, are said to be homotopic if there exists an exact $C^{\prime}$-algebra $B$ with two augmentations $s_{0}, s_{1}$ and a morphism of $C^{\prime}$-algebras $\Phi: A \rightarrow A^{\prime} \otimes_{C^{\prime}} B$ such that $\phi_{i}=\left(1 \otimes s_{i}\right) \Phi, i=0,1$, and $\left(\epsilon_{A^{\prime}} \otimes 1\right) \Phi$ is the composition

$$
A \stackrel{\epsilon_{A}}{\rightarrow} K^{\langle 1\rangle} \rightarrow B .
$$


(This is an algebraic analogy of a base point preserving homotopy. See [2].)

TheOREM. If $\phi_{0}$ and $\phi_{1}$ are homotopic, the $\pi^{1}\left(\phi_{0}\right)=\pi^{1}\left(\phi_{1}\right)$.

\section{BiBLIOGRAPHY}

1. K.-T. Chen, Algebraic paths, J. Algebra 10 (1968), 8-36.

2. - Homotopy of algebras, J. Algebra 10 (1968), 183-193.

3. - Covering-space-like algebras, J. Algebra (to appear).

4. S. MacLane, Categorical algebra, Bull. Amer. Math. Soc. 71 (1965), 40-106.

University of IllinoIs, URbana, IllinoIs 61801 